\title{
Local critical behaviour at aperiodic surface extended perturbation in the Ising quantum chain
}

\author{
Dragi Karevski, Gábor Palágyi† and Loïc Turban \\ Laboratoire de Physique du Solideł, Université Henri Poincaré (Nancy I), BP 239, \\ F-54506 Vandœuvre lès Nancy Cedex, France
}

\begin{abstract}
The surface critical behaviour of the semi-infinite one-dimensional quantum Ising model in a transverse field is studied in the presence of an aperiodic surface extended modulation. The perturbed couplings are distributed according to a generalized Fredholm sequence, leading to a marginal perturbation and varying surface exponents. The surface magnetic exponents are calculated exactly whereas the expression of the surface energy density exponent is conjectured from a finitesize scaling study. The system displays surface order at the bulk critical point, above a critical value of the modulation amplitude. It may be considered as a discrete realization of the Hilhorst-van Leeuwen model.
\end{abstract}

\section{Introduction}

The influence of bulk quasiperiodic or aperiodic perturbations on the critical properties at second order phase transitions has been an active field of research during the last years. Different problems were studied numerically on the two-dimensional Penrose lattice including the Ising model (Godrèche et al 1986, Okabe and Niizeki 1988, Sørensen et al 1991), percolation (Sakamoto et al 1989, Zhang and De'Bell 1993) and the self-avoiding-walk (Langie and Iglói 1992). In all these cases, no change in the critical exponents was observed. Universality was also preserved for threedimensional quasiperiodic systems (Okabe and Niizeki 1990). On the contrary, a continuously varying roughness exponent was obtained for interface roughening in two dimensions with a modulation of the couplings following the Fibonacci sequence (Henley and Lipowsky 1987, Garg and Levine 1987).

The aperiodically layered two-dimensional Ising model has been also extensively studied (Iglói 1988, Doria and Satija 1988, Benza 1989, Ceccato 1989a, 1989b, Henkel and Patkós 1992, Lin and Tao 1990,1992a,1992b, You et al 1992, Turban and Berche 1993) following earlier pioneering works on randomly or arbitrarily layered Ising systems (McCoy and $\mathrm{Wu} 1968, \mathrm{Au}-$ Yang and McCoy 1974). The problem was mainly treated in the extreme anisotropic limit where the constant intralayer interaction $K_{1}=J_{1} / k_{B} T$ goes to infinity while the modulated interlayer interactions $K_{2}(k)$ go to zero, keeping fixed the ratio $\lambda_{k}=K_{2}(k) / K_{1}^{*}$ where $K_{1}^{*}$ is related to $K_{1}$ through

$\dagger$ Permanent address: Department of Theoretical Physics, University of Szeged, H-6720 Szeged, Hungary

$\ddagger$ Unité de Recherche Associée au CNRS No 155

cond-mat/9411030 
duality (Kogut 1979). In this limit, the physics of the system is governed by the one-dimensional quantum Ising model (QIM) with the Hamiltonian

$$
\mathcal{H}=-\frac{1}{2} \sum_{k=1}^{\infty}\left[\sigma_{k}^{z}+\lambda_{k} \sigma_{k}^{x} \sigma_{k+1}^{x}\right]
$$

Some exact results have been obtained with Fibonacci, Thue-Morse and other aperiodic modulations for which the critical behaviour is universal. In other cases the Onsager logarithmic singularity of the specific heat was found to be washed out (Tracy 1988), like in the random McCoy-Wu model. The structure of the critical excitation spectrum and the related conformal aspects have been also explored (Iglói 1988, Grimm and Baake 1994).

The situation was recently clarified through the introduction of a relevanceirrelevance criterion (Luck 1993a, 1993b, Iglói 1993) generalizing to aperiodic systems the Harris criterion for random systems (Harris 1974). The cumulated deviation from the average coupling $\bar{\lambda}, \Delta(L)=\sum_{k=1}^{L}\left(\lambda_{k}-\bar{\lambda}\right)$, scales with the chain length $L$ as $\delta L^{\omega}$ where $\delta$ is the amplitude of the modulation and $\omega$ is the wandering exponent of the aperiodic sequence related to the leading eigenvalues of its substitution matrix (Queffelec 1987, Dumont 1990). Under a change of the length scale by a factor $b=L / L^{\prime}$, the average thermal perturbation $\Delta(L) / L$ is multiplied by $b^{1 / \nu}$, where $\nu$ is the bulk correlation length exponent, and the amplitude transforms as

$$
\delta^{\prime}=b^{\Phi / \nu} \delta, \quad \Phi=1+\nu(\omega-1) .
$$

The relevance of the aperiodic perturbation depends on the sign of the crossover exponent $\Phi$. In the two-dimensional Ising model with $\nu=1$ the modulation is irrelevant when $\omega<0$, i. e. for the much studied Fibonacci and Thue-Morse sequences, marginal when $\omega=0$ and relevant when $\omega>0$. One expects varying exponents In the marginal case and a new type of critical behaviour in the relevant case.

The three types of critical behaviour were indeed obtained in recent exact calculations of the surface magnetization of the QIM (Turban, Iglói and Berche 1994, Iglói and Turban 1994, Turban, Berche and Berche 1994).

In the present work, we study surface aperiodic perturbations generated through the Fredhom sequence (Dekking et al 1983) and its generalizations. Such sequences lead to a vanishing density of defects in the bulk of the system and the bulk critical properties are left unchanged. On the other hand, they induce a marginal surface extended perturbation so that the surface critical exponents are nonuniversal, varying with the modulation amplitude. Such aperiodic perturbations may be considered as discrete realizations of the Hilhorst-van Leeuwen model (Hilhorst and van Leeuwen 1981, see also Iglói, Peschel and Turban 1993, for a recent review).

The properties of the generalized Fredholm sequence are studied in section 2 . The surface magnetization is calculated exactly in section 3 and the surface energy exponent is obtained through finite-size scaling in section 4 . The results are discussed in the last section.

\section{Generalized Fredholm sequence}

We consider a generalized Fredholm sequence generated through substitution on the three letters $A, B$ and $C$ 


$$
\begin{aligned}
A \rightarrow \mathcal{S}(A) & =A B C C \cdots C \\
B \rightarrow \mathcal{S}(B) & =B C C C \cdots C \\
C \rightarrow \mathcal{S}(C) & =\underbrace{C C C C \cdots C}_{m}
\end{aligned}
$$

which is the characteristic sequence of the powers of $m$. With words of length $m=2$, one recovers the usual Fredholm sequence (Dekking et al 1983).

The substitution matrix $\mathbf{M}$, with entries giving the numbers of $A, B$ and $C$ in $\mathcal{S}(A), \mathcal{S}(B)$ and $\mathcal{S}(C)$, then reads

$$
\mathbf{M}=\left(\begin{array}{ccc}
1 & 0 & 0 \\
1 & 1 & 0 \\
m-2 & m-1 & m
\end{array}\right)
$$

with eigenvalues $\Omega_{1}=m, \Omega_{2,3}=1$. The wandering exponent is given by

$$
\omega=\frac{\ln \Omega_{2}}{\ln \Omega_{1}}=0
$$

so that, according to equation (1.2), the Fredholm modulation is a marginal perturbation for the QIM. The numbers of letters of each type at the $n$th step in the inflation process are given by the matrix elements of $\mathbf{M}^{\mathbf{n}}$. The asymptotic letter densities $\rho_{A}, \rho_{B}$ and $\rho_{C}$ are related to the components of the right eigenvector associated with the largest eigenvalue $\Omega_{1}$ with here

$$
\rho_{A}=0, \quad \rho_{B}=0, \quad \rho_{C}=1
$$

independent of $m$.

In the following, the couplings in the Hamiltonian (1.1), which are distributed according to the aperiodic sequence, will be written as $\lambda_{k}=\lambda r^{f_{k}}$ where $\lambda$ is the unperturbed (bulk) interaction and $r$ characterizes the modulation. We associate an unperturbed coupling $\lambda$, i. e. $f_{k}=0$, to the letters $A$ or $C$ and a perturbed coupling $\lambda r, f_{k}=1$, to $B$. For example, starting on $A$ with $m=2$, one obtains the following sequences after $n$ iterations:

$$
\begin{array}{ccccccccccccccccccc}
n=0 & A & & & & & & & & & & & & & & & \\
n=1 & A & B & & & & & & & & & & & & & & \\
n=2 & A & B & B & C & & & & & & & & & & & & \\
n=3 & A & B & B & C & B & C & C & C & & & & & & & & \\
n=4 & A & B & B & C & B & C & C & C & B & C & C & C & C & C & C & C \\
f_{k} & 0 & 1 & 1 & 0 & 1 & 0 & 0 & 0 & 1 & 0 & 0 & 0 & 0 & 0 & 0 & 0
\end{array}
$$

Equation (2.1) leads to the following relations for the $f_{k} \mathrm{~s}$ :

$$
\begin{array}{ll}
f_{m p+1}=f_{p+1}, & \\
f_{m p+2}=0 & (p>0), \quad f_{2}=1, \\
f_{m p+q}=0 & (q=3,4, \ldots, m) .
\end{array}
$$


They can be used to deduce similar recursion relations for the number of perturbed couplings $n_{j}=\sum_{k=1}^{j} f_{k}$ in a sequence with length $j$, which are obtained by splitting the sum over $k$ into $m$ sums over $p$, giving:

$$
\begin{aligned}
& n_{m l+1}=n_{l+1}+1 \quad(l>0), \quad n_{1}=0, \\
& n_{m l+q}=n_{l+1}+1 \quad(q=2,3, \ldots, m) \text {. }
\end{aligned}
$$

Iterating these relations, one may check that $f_{k}=1$ when $k=m^{l}+1(l=0,1,2, \ldots)$. For a sequence with length $L=m^{l}$,

$$
n_{L}=l=\frac{\ln L}{\ln m},
$$

and the asymptotic density of defects satisfies

$$
\rho_{\infty}=\lim _{L \rightarrow \infty} \frac{n_{L}}{L}=\rho_{B}=0,
$$

i. e. the generalized Fredholm modulation introduces an extended surface perturbation in the system.

\section{Surface magnetization}

The surface magnetization $m_{s}$ follows from the asymptotic behaviour of the surface spin-spin correlation function, $\lim _{t \rightarrow \infty}\left\langle\sigma_{1}^{x}(0) \sigma_{1}^{x}(t)\right\rangle$, which gives the square of this quantity on a semi-infinite system. Writing the correlation function in the basis which diagonalizes the Hamiltonian in equation (1.1), $m_{s}$ can be expressed as the matrix element $\left\langle\sigma\left|\sigma^{x}\right| 0\right\rangle$, where $|0\rangle$ is the groundstate and $|\sigma\rangle$ the first excited state of $\mathcal{H}$ (Schultz et al 1964). These two states become degenerate in the ordered phase $\lambda>\lambda_{c}$ as a consequence of long-range order.

The Hamiltonian can be put in diagonal form (Lieb et al 1961)

$$
\mathcal{H}=\sum_{\nu} \epsilon_{\nu}\left(\eta_{\nu}^{\dagger} \eta_{\nu}-\frac{1}{2}\right)
$$

using the Jordan-Wigner transformation (Jordan and Wigner 1928) followed by a canonical transformation to the diagonal fermion operators $\eta_{\nu}$. The fermion excitation spectrum is obtained as the solution of the eigenvalue problem

$$
\begin{aligned}
& \epsilon_{\nu} \psi_{\nu}(k)=-\phi_{\nu}(k)-\lambda_{k} \phi_{\nu}(k+1) \\
& \epsilon_{\nu} \phi_{\nu}(k)=-\lambda_{k-1} \psi_{\nu}(k-1)-\psi_{\nu}(k)
\end{aligned}
$$

where the $\phi_{\nu}(k)$ and $\psi_{\nu}(k)$ are the components of two normalized eigenvectors which satisfy the boundary conditions $\phi_{\nu}(0)=\psi_{\nu}(0)=0$.

Rewriting $\sigma^{x}$ in terms of diagonal fermions with $|\sigma\rangle=\eta_{1}^{\dagger}|0\rangle$, it can be shown that $m_{s}$ is also given by the first component $\phi_{1}(1)$ of the eigenvector corresponding to the smallest excitation. According to the first equation in (3.2), in the ordered phase where $\epsilon_{1}$ vanishes, other components of the eigenvector follow from the recursion relation

$$
\phi_{1}(k+1)=-\lambda_{k}^{-1} \phi_{1}(k) .
$$


The normalization of the eigenvector then leads to the surface magnetization (Peschel 1984)

$$
m_{s}=\left(1+\sum_{j=1}^{\infty} \prod_{k=1}^{j} \lambda_{k}^{-2}\right)^{-1 / 2}
$$

For the aperiodic system, with $\lambda_{k}=\lambda r^{f_{k}}$, this leads to:

$$
m_{s}=[S(\lambda, r)]^{-1 / 2}, \quad S(\lambda, r)=\sum_{j=0}^{\infty} \lambda^{-2 j} r^{-2 n_{j}}, \quad n_{0}=0
$$

The critical coupling $\lambda_{c}$ generally follows from (Pfeuty 1979):

$$
\lim _{j \rightarrow \infty} 1 / j \sum_{k=1}^{j} \ln \lambda_{k}=0
$$

Here, $\lambda_{c}=r^{-\rho_{\infty}}=1$ keeps its unperturbed value, as expected for an extended surface perturbation.

In order to calculate the sum in (3.5) let us rewrite it as

$$
S(\lambda, r)=1+\lambda^{-2}+T(\lambda, r), \quad T(\lambda, r)=\sum_{j=2}^{\infty} \lambda^{-2 j} r^{-2 n_{j}} .
$$

The second sum can be splitted into $m$ parts as

$$
\begin{aligned}
T(\lambda, r)= & \sum_{l=1}^{\infty} \lambda^{-2(m l+1)} r^{-2 n_{m l+1}}+\sum_{l=0}^{\infty} \lambda^{-2(m l+2)} r^{-2 n_{m l+2}}+\cdots \\
& +\sum_{l=0}^{\infty} \lambda^{-2(m l+m)} r^{-2 n_{m l+m}}
\end{aligned}
$$

and, using (2.7), the following functional equation is obtained:

$$
T(\lambda, r)=\frac{r^{-2}}{\lambda^{2}-1}\left[\lambda^{-2}-\lambda^{-2 m}+\left(\lambda^{2 m}-1\right) T\left(\lambda^{m}, r\right)\right] .
$$

This can be iterated to give:

$$
T(\lambda, r)=\sum_{l=0}^{\infty} r^{-2(l+1)} \lambda^{-2 m^{l+1}} \sum_{p=0}^{(m-1) m^{l}-1} \lambda^{2 p}
$$

The critical behaviour can be extracted, applying a finite-size scaling method due to Iglói. Assuming that the surface magnetization displays a power-law singularity with a critical exponent $\beta_{s}, S(\lambda, r)$ behaves as $t^{-2 \beta_{s}}$ with $t=\lambda_{c}^{-2}-\lambda^{-2}$, near the critical point $\lambda_{c}=1$. It can be shown (Iglói 1986) that the sum $S_{L}$ of the first $L$ terms in a power series expansion in $\lambda^{-2}$ asymptotically scales like $L^{2 \beta_{s}}$ at the critical point. 


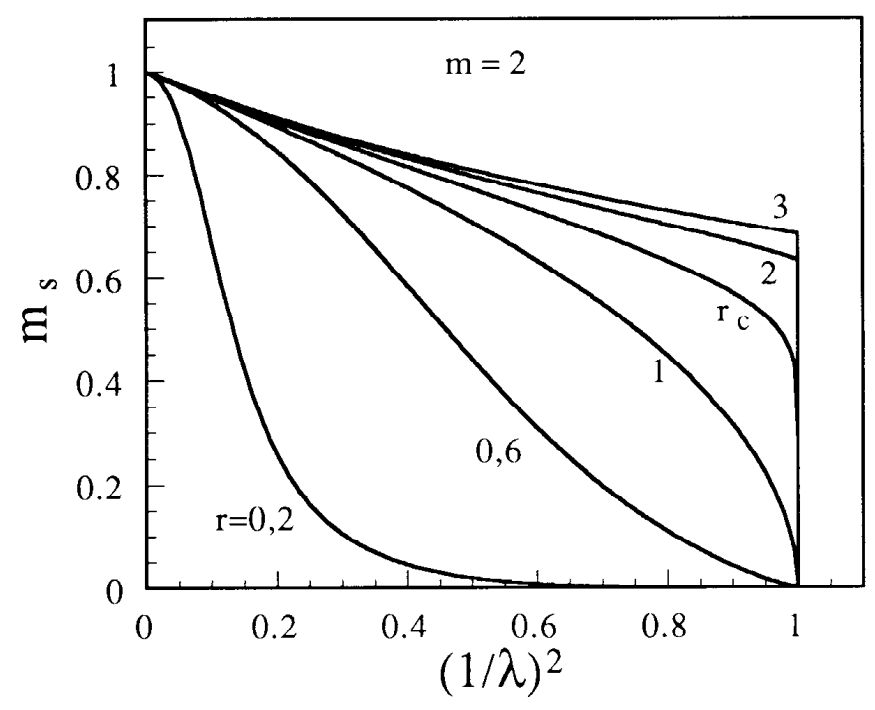

Figure 1. Spontaneous surface magnetization $m_{s}$ of the Ising quantum chain with the $m=2$ Fredholm aperiodic modulation as a function of the square of the reduced coupling $\lambda_{c} / \lambda$ for different values of the coupling ratio $r$. The surface transition is first-order when $r>r_{c}=\sqrt{2}$.

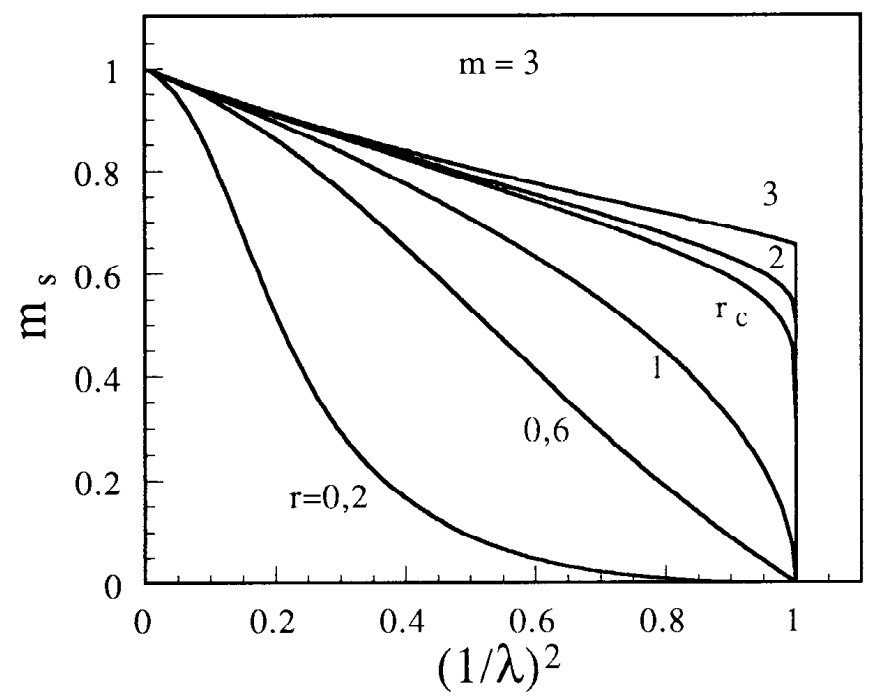

Figure 2. Spontaneous surface magnetization $m_{s}$ of the Ising quantum chain with the $m=3$ Fredholm aperiodic modulation as a function of the square of the reduced coupling $\lambda_{c} / \lambda$ for different values of the coupling ratio $r$. The surface transition is first-order when $r>r_{c}=\sqrt{3}$.

One easily verifies that the term $l=n$ of the sum in (3.10) contains the powers of $\lambda^{-2}$ from $m^{n}+1$ to $m^{n+1}$. Cutting the sum at $n-1$, one collects the contribution to the first $L=m^{n}$ terms of the series expansion and, using (3.7), 


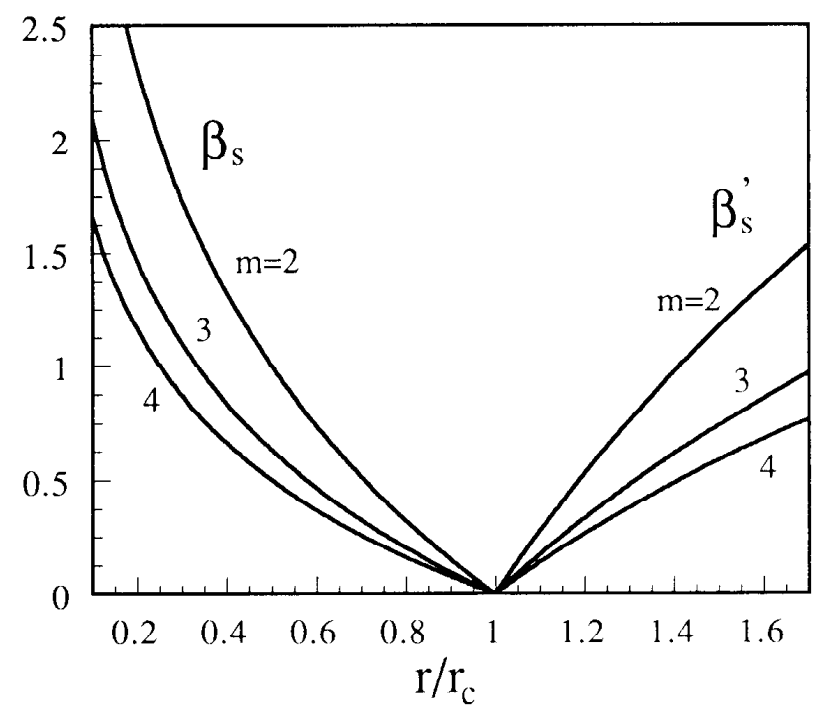

Figure 3. Ising Surface magnetic exponents $\beta_{s}$ and $\beta_{s}^{\prime}$ versus reduced coupling ratio $r / r_{c}$ for the generalized Fredholm sequence with $m=2,3,4$. The exponent $\beta_{s}^{\prime}$ is associated with the approach towards the critical surface magnetization when $r>r_{c}$.

$S_{L=m^{n}}(1, r)=2+\frac{m-1}{r^{2}} \sum_{l=0}^{n-1}\left(\frac{m}{r^{2}}\right)^{l}=2+\frac{m-1}{r^{2}}\left[\frac{1-\left(m r^{-2}\right)^{n}}{1-m r^{-2}}\right]$.

Two regimes have then to be considered: when $r<r_{c}=\sqrt{m}, S_{L=m^{n}}$ behaves as $\left(m r^{-2}\right)^{n}$ whereas it is $O(1)$ when $r>r_{c}$. It follows that

$$
\begin{array}{ll}
\beta_{s}=\frac{1}{2}-\frac{\ln r}{\ln m}, & r \leq r_{c}=\sqrt{m}, \\
\beta_{s}=0, & r>r_{c} .
\end{array}
$$

When $r \leq r_{c}$ the surface transition is second order as shown in figures 1 and 2 . The exponent $\beta_{s}$ depends on the modulation amplitude, as expected with a marginal perturbation. This dependence is shown in figure 3 . The exponent goes to zero linearly at $r_{c}$ as $\left(r_{c}-r\right) /\left(2 r_{c} \ln r_{c}\right)$.

The vanishing of $\beta_{s}$ for $r>r_{c}$ signals the occurence of surface order at the bulk critical point. Since the surface is one-dimensional in the corresponding layered twodimensional classical system, it cannot stay ordered when the bulk disorders and the surface transition is first-order in this regime as shown in figures 1 and 2 . The existence of surface order at $\lambda_{c}$ and above is linked to the localization of the eigenvector $\phi_{1}$. It remains normalizable even at the critical point when $r>r_{c}$.

The value of the critical surface magnetization $m_{s, c}$ follows from (3.5) taking the limit $n \rightarrow \infty$ in (3.11), which gives:

$$
m_{s, c}=\sqrt{\frac{r^{2}-m}{2 r^{2}-m-1}}, \quad r \geq r_{c} .
$$




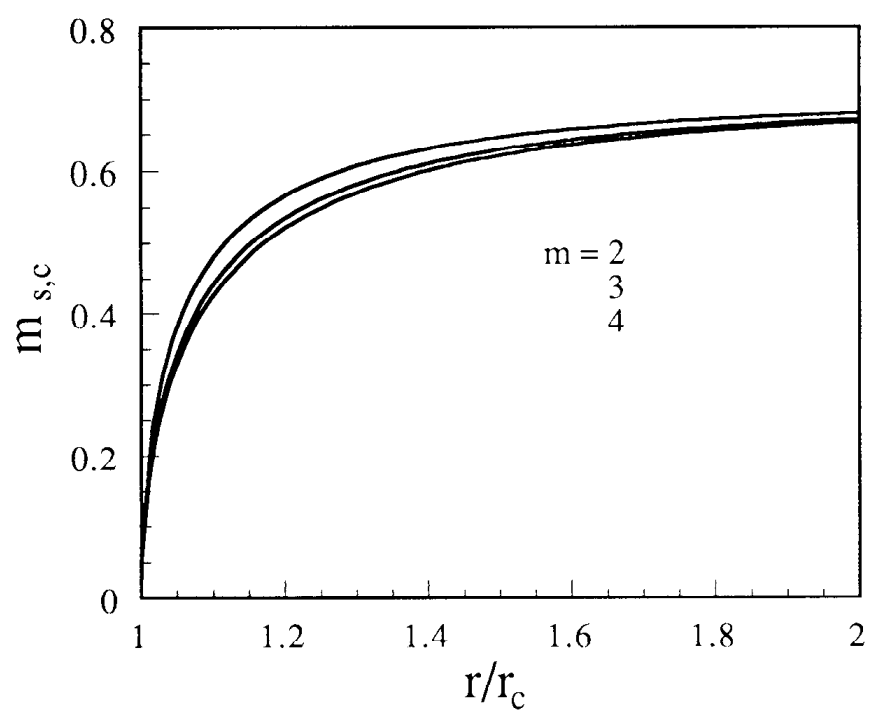

Figure 4. Variation of the critical surface magnetization $m_{s, c}$ with the reduced coupling ratio $r / r_{c}$ for the generalized Fredholm sequence with $m=2,3,4$ from top to bottom.

The behaviour of $m_{s, c}$ is shown in figure 4 . It vanishes with a square-root singularity at $r_{c}$.

The approach to the critical surface magnetization above $r_{c}$ involves another surface exponent $\beta_{s}^{\prime}$ such that $m_{s}-m_{s, c} \sim t^{\beta_{s}^{\prime}}$. It may be calculated, using the same method as above, by considering the series expansion $S_{L}^{\prime}(1, r)=S_{L}(1, r)-\left[m_{s, c}\right]^{-2}$, with $L=m^{n}$, which scales like $L^{-\beta_{s}^{\prime}}$. One obtains:

$$
\begin{aligned}
& S_{L}^{\prime}(1, r)=\frac{m-1}{r^{2}} \sum_{l=n}^{\infty}\left(\frac{m}{r^{2}}\right)^{l} \sim\left(\frac{m}{r^{2}}\right)^{n} \sim m^{-n \beta_{s}^{\prime}}, \\
& \beta_{s}^{\prime}=2 \frac{\ln r}{\ln m}-1 .
\end{aligned}
$$

The exponent $\beta_{s}^{\prime}$, shown in figure 3 , vanishes linearly at $r_{c}$ as $\left(r-r_{c}\right) /\left(r_{c} \ln r_{c}\right)$.

At the critical value of the modulation amplitude, according to (3.11), the finite series expansion gives

$$
S_{L=m^{n}}\left(1, r_{c}\right)=2+\frac{m-1}{m} n \sim \ln L,
$$

so that the surface magnetization vanishes there with a logarithmic singularity

$$
m_{s}\left(r_{c}\right) \sim(-\ln t)^{-1 / 2}
$$

The variation of $m_{s}\left(r_{c}\right)$ with $\lambda^{-2}$ is shown in figures 1 and 2 . 


\section{Surface energy}

The critical behaviour of the surface energy can be studied by considering the finitesize behaviour of the matrix element $e_{s}=\left\langle\varepsilon\left|\sigma_{1}^{z}\right| 0\right\rangle$ which does not contain any regular part and scales like $L^{-x_{e_{s}}}$ where $x_{e_{s}}$ is the dimension of the surface energy density. Here, the state $|\varepsilon\rangle$ is the lowest two-fermion eigenstate $\eta_{1}^{\dagger} \eta_{2}^{\dagger}|0\rangle$ of the Hamiltonian (3.1). Writing $\sigma_{1}^{z}$ in terms of diagonal fermions, one obtains

$$
e_{s}=\psi_{1}(1) \phi_{2}(1)-\phi_{1}(1) \psi_{2}(1)=\left(\epsilon_{2}-\epsilon_{1}\right) \phi_{1}(1) \phi_{2}(1)
$$

where, in the last expression, we used the relation $\psi_{\nu}(1)=-\epsilon_{\nu} \phi_{\nu}(1)$ which follows from the second equation in (3.2) for the surface components of the eigenvectors.

The matrix element $e_{s}$ on finite systems with size $L$ of the form $m^{n}$ was obtained through a numerical solution of the eigenvalue problem

$$
\begin{aligned}
& \lambda_{k-1} \phi_{\nu}(k-1)+\left(\lambda_{k-1}^{2}+1\right) \phi_{\nu}(k)+\lambda_{k} \phi_{\nu}(k+1)=\epsilon_{\nu}^{2} \phi_{\nu}(k), \\
& \phi_{\nu}(1)+\lambda_{1} \phi_{\nu}(2)=\epsilon_{\nu}^{2} \phi_{\nu}(1), \quad \phi_{\nu}(L+1)=0 .
\end{aligned}
$$

For small chains $\left(L \leq 2^{15}\right.$ or $\left.3^{9}\right)$, the complete excitation matrix was diagonalized while for longer chains (up to $L=2^{17}$ or $3^{10}$ ), equation (4.2) was rewritten as a matrix recursion relation

$$
\left(\begin{array}{c}
\phi_{\nu}(k+1) \\
\phi_{\nu}(k)
\end{array}\right)=\left(\begin{array}{cc}
\frac{\epsilon_{\nu}^{2}-\lambda_{k-1}^{2}-1}{\lambda_{k}} & -\frac{\lambda_{k-1}}{\lambda_{k}} \\
1 & 0
\end{array}\right)\left(\begin{array}{c}
\phi_{\nu}(k) \\
\phi_{\nu}(k-1)
\end{array}\right)
$$

and varying $\epsilon_{\nu}$, we looked for the zeros of $\phi_{\nu}(L+1)$, with $\phi_{\nu}(0)=0$ and $\phi_{\nu}(1)$ arbitrary in the first vector. Once the zeros corresponding to the two lowest eigenvalues are found, the corresponding eigenvectors are normalized in order to evaluate (4.1). The exponent $x_{e_{s}}$ is then deduced from the slope at large $L$ in a log-log plot. The results are shown in figures 5 and 6 .

The behaviour of $x_{e_{s}}$ below $r_{c}$ can be deduced from finite-size scaling considerations. Low-energy excitations scale as $L^{-1}$ and due to the factor $\epsilon_{\nu}$, the l. h. s. of the first equation in (3.2) can be neglected. The leading finite-size behaviours of $\phi_{1}(1)$ and $\phi_{2}(1)$ are the same and follow from (3.4) by cutting the sum at $j=L$. The calculation proceeds like for $\beta_{s}$ and gives $\phi_{1}(1) \sim \phi_{2}(1) \sim L^{-x_{m_{s}}}$ with $x_{m_{s}}=\beta_{s}=1 / 2-\ln r / \ln m$. As a by-product, we recover $\nu=\beta_{s} / x_{m_{s}}=1$ for the bulk correlation length exponent, as expected for a surface perturbation. Collecting these results in (4.1), we obtain:

$$
e_{s}(L) \sim L^{-1-2 \beta_{s}}, \quad x_{e_{s}}=2-2 \frac{\ln r}{\ln m}, \quad r \leq r_{c} .
$$

When $r>r_{c}$ one finds different size-dependences for the first and second excitations as well as for the corresponding components of the eigenvector. Due to surface ordering, the first excitation vanishes anomalously as $L^{-2 \ln r / \ln m}$, i. e. quicker than the higher ones with the usual $L^{-1}$ behaviour. Equation (3.3) can still be used to calculate the size-dependence of $\phi_{1}(1)$ which, following the same steps as in the last section, gives $m_{s}(L)=$ constant term $+L^{-x_{m_{s}}^{\prime}}$ where $x_{m_{s}}^{\prime}=\beta_{s}^{\prime}$. The term on the l. h.s. 


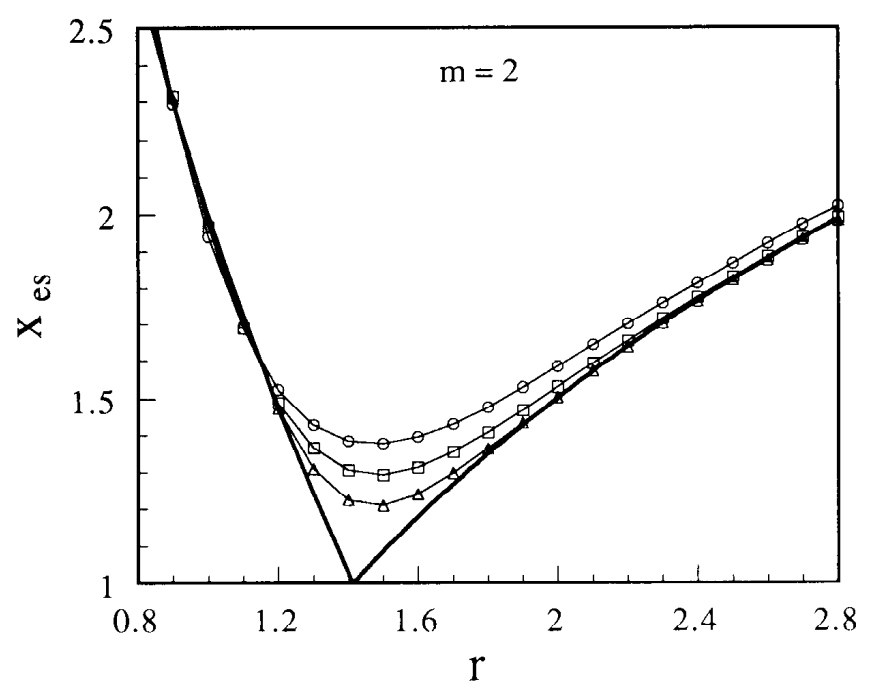

Figure 5. Surface energy density exponent as a function of the coupling ratio $r$ for the Fredholm sequence with $m=2$. The points correspond to finite-size scaling results on chains with sizes of the form $2^{n}$ up to $L=2^{10}, 2^{12}, 2^{17}$ from top to bottom. The heavy line gives the conjectured analytical result.

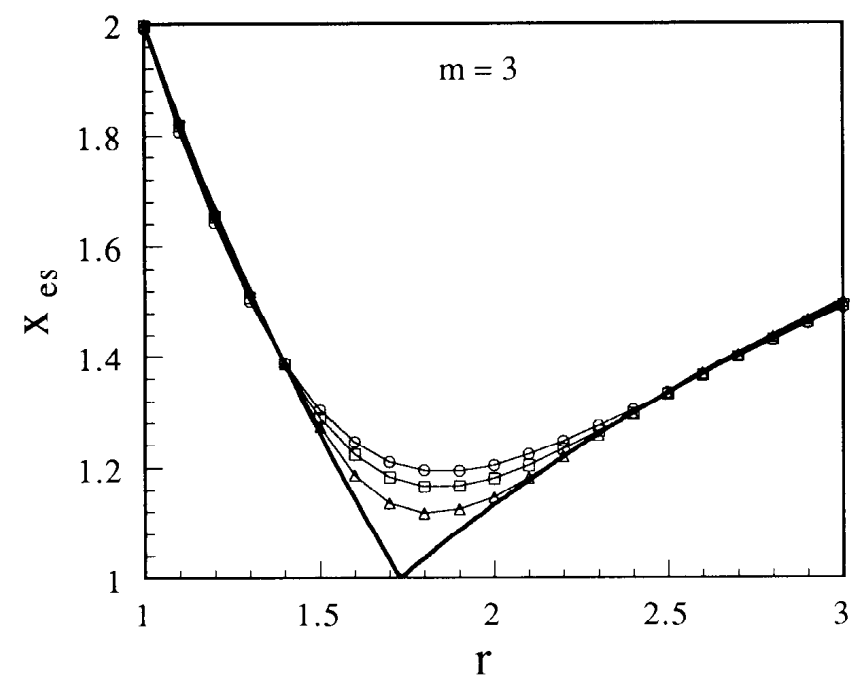

Figure 6. Surface energy density exponent as a function of the coupling ratio $r$ for the generalized Fredholm sequence with $m=3$. The points correspond to finite-size scaling results on chains with sizes of the form $3^{n}$ up to $L=3^{7}, 3^{8}, 3^{9}$ from top to bottom. The heavy line gives the conjectured analytical result.

in (3.2) can no longer be neglected for $\phi_{2}(1)$. The size-dependence $L^{1 / 2-\ln r / \ln m}$ was obtained numerically. Together, this gives:

$$
e_{s}(L) \sim L^{-1-x_{m_{s}}^{\prime} / 2}, \quad x_{e_{s}}=\frac{1}{2}+\frac{\ln r}{\ln m}, \quad r>r_{c} .
$$


One may notice that the anomalous behaviour of the first excitation is in agreement with a scaling theory for first-order line transitions involving an irrelevant variable and leading to a $L^{1-2 x_{e s}}$ size-dependence (Iglói and Turban 1993). Numerical results for the surface exponent $x_{e_{s}}$ in figures 5 and 6 , converge smoothly with increasing size towards the conjectured analytic expressions.

\section{Discussion}

As already mentioned in the introduction, the aperiodic surface extended perturbation treated here, displays some similarities with the Hilhorst-van Leeuwen model (Hilhorst and van Leeuwen 1981) in which the coupling, at a distance $k$ from a free surface, takes the form

$$
\lambda_{k}=\lambda\left(1+\frac{\alpha}{k^{y}}\right)
$$

For the Ising model in $1+1$ dimensions, the marginal case corresponds to a decay exponent $y=1$. The critical surface order appears at $\alpha_{c}=1 / 2$ and the surface critical exponents have the following dependence on the perturbation amplitude:

$$
\begin{aligned}
& \beta_{s}=x_{m_{s}}=\frac{1}{2}-\alpha, \quad x_{e_{s}}=2-2 \alpha, \quad \alpha \leq \alpha_{c}, \\
& \beta_{s}^{\prime}=x_{m_{s}}^{\prime}=2 \alpha-1, \quad x_{e_{s}}=\frac{1}{2}+\alpha, \quad \alpha>\alpha_{c} .
\end{aligned}
$$

A comparison with previous results in equations (3.12), (3.14), (4.4) and (4.5) shows that the exponents of the aperiodic system are recovered through the following correspondence:

$$
\alpha \rightarrow \frac{\ln r}{\ln m}
$$

In the Hilhorst-van Leeuwen model, the integrated relative perturbation at a distance $L$ is given by:

$$
\sum_{k=1}^{L} \frac{\lambda_{k}-\lambda}{\lambda}=\alpha \sum_{k=1}^{L} \frac{1}{k} \simeq \alpha \ln L .
$$

On the average, the same logarithmic dependence is found with the aperiodic perturbation for which, on a chain with length $L=m^{n}$ according to (2.8), the corresponding quantity is

$$
\sum_{k=1}^{L} \frac{\lambda_{k}-\lambda}{\lambda}=(r-1) n_{L}=\frac{r-1}{\ln m} \ln L .
$$

This would suggest the identification $\alpha=(r-1) / \ln m$ which, actually, is only valid up to the first order in $r-1$, i. e. for a weak modulation. The fluctuations around the average logarithmic behaviour modify this expression for a stronger perturbation. 
The correct identification can be obtained by considering the finite-size behaviour of the critical coupling which follows from equation (3.6) with

$$
\ln \left[\lambda_{c}(L)\right]=-\ln r \frac{n_{L}}{L}=-\frac{\ln r}{\ln m} \frac{\ln L}{L}
$$

for the aperiodic system, whereas

$$
\ln \left[\lambda_{c}(L)\right]=-\frac{1}{L} \sum_{k=1}^{L} \ln \left(1+\frac{\alpha}{k}\right) \simeq-\alpha \frac{\ln L}{L}
$$

in the Hilhorst-van Leeuwen model.

To conclude, one may notice that an experimental realization of the Hilhorst-van Leeuwen model with its $1 / k^{y}$ decay of the couplings appears difficult while its aperiodic counterpart, studied here in the marginal case, could be more easily obtained (at least in three dimensions) using appropriate sequences in multi-layer systems.

\section{Acknowledgments}

LT is indebted to Ferenc Iglói for stimulating discussions. DK and GP thank Bertrand Berche for help in the numerical work which was supported by CNIMAT under project No 155C93b.

\section{References}

Au-Yang H and McCoy B M 1974 Phys. Rev. B 10886

Benza G V 1989 Europhys. Lett. 8321

Ceccato H A 1989a Phys. Rev. Lett. 62203

Ceccato H A 1989b Z. Phys. B 75253

Dekking M, Mendès-France M and van der Poorten A 1983 Math. Intelligencer 4130

Doria M M and Satija I I 1988 Phys. Rev. Lett. 60444

Dumont J M 1990 Number Theory and Physics, Springer Proc. Phys. vol 47 ed. J M Luck, P Moussa and M Waldschmidt (Berlin: Springer) p 185

Garg A and Levine D 1987 Phys. Rev. Lett. 591683

Godrèche C, Luck J M and Orland H J 1986 J. Stat. Phys. 45777

Grimm U and Baake M 1994 J. Stat. Phys. 741233

Harris AB 1974 J. Phys. C: Solid State Phys. 71671

Henkel M and Patkós A 1992 J. Phys. A: Math. Gen. 255223

Henley C L and Lipowsky R 1987 Phys. Rev. Lett. 591679

Hilhorst H J and van Leeuwen J M J 1981 Phys. Rev. Lett. 471188

Iglói F 1986 J. Phys. A: Math. Gen. 193077

1988 J. Phys. A: Math. Gen. 21 L911 1993 J. Phys. A: Math. Gen. 26 L703

Iglói F, Peschel I and Turban L 1993 Adv. Phys. 42683

Iglói F and Turban L 1993 Phys. Rev. B 473404

1994 Europhys. Lett. 2791

Jordan P and Wigner E 1928 Z. Phys. 47631

Kogut J 1979 Rev. Mod. Phys. 51659

Langie G and Iglói F 1992 J. Phys. A: Math. Gen. 25 L487

Lieb E H, Schultz T D and Mattis D C 1961 Ann. Phys., NY 16406

Lin Z and Tao R 1990 Phys. Lett. 150A 11

— 1992a J. Phys. A: Math. Gen. 252483 
1992b Phys. Rev. B 4610808

Luck J M 1993a J. Stat. Phys. 72417

1993b Europhys. Lett. 24359

McCoy B M and Wu T T 1968 Phys. Rev. 176631

Okabe Y and Niizeki K 1988 J. Phys. Soc. Japan 571536

1990 J. Phys. A: Math. Gen. 23 L733

Peschel I 1984 Phys. Rev. B 306783

Pfeuty P 1979 Phys. Lett. 72A 245

Queffélec M 1987 Substitution Dynamical Systems-Spectral Analysis, Lecture Notes in Mathematics vol 1294 ed. A Dold and B Eckmann (Berlin: Springer) p 97

Sakamoto S, Yonezawa F, Aoki K, Nosé S and Hori M 1989 J. Phys. A: Math. Gen. 22 L705

Schultz T D, Mattis D C and Lieb E H 1964 Rev. Mod. Phys. 36856

Sørensen E S, Jarić M V and Ronchetti M 1991 Phys. Rev. B 449271

Tracy C A 1988 J. Phys. A: Math. Gen. 21 L603

Turban L and Berche B 1993 Z. Phys. B 92307

Turban L, Iglói F and Berche B 1994 Phys. Rev. B 4912695

Turban L, Berche P E and Berche B 1994 J. Phys. A: Math. Gen. 276349

You J Q, Yan J R and Zhong J X 1992 J. Math. Phys. 333901

Zhang C and De'Bell K 1993 Phys. Rev. B 478558 